\title{
COMBINED GAUSSIAN FILTERING, DWT AND MORPHOLOGICAL APPROACH FOR SATELLITE IMAGE ENHANCEMENT
}

\author{
Ch.Haritha $^{1}$, B.Rupavani ${ }^{2}$, P.Lakshmi Prasanna ${ }^{3}$, A.Venkataramana ${ }^{4}$, Ch.Rajendra Prasad ${ }^{5}$ \\ ${ }^{1}$ Student in ECE, Sreenidhi institute of science and Technology, Hyderabad-5000036, Telangana, India \\ ${ }^{2}$ Student in ECE, Sreenidhi institute of science and Technology, Hyderabad-5000036, Telangana, India \\ ${ }^{3}$ Student in ECE, Sreenidhi institute of science and Technology, Hyderabad-5000036, Telangana, India \\ ${ }^{4}$ Lecturer in ECE, Q.Q. Government Polytechnic, Old city, Hyderabad-500064, Telangana, India \\ ${ }^{5}$ Head of the department in ECE, Government Polytechnic, MEDHAK-520110, Telangana, India
}

\begin{abstract}
In this paper, a method for satellite image enhancement using Gaussian filtering, DWT and morphological filtering is proposed. Gaussian filtering is applied for noise removal of the image. The DWTt is used to transform image based on frequency. Morphological filtering is used for retaining the originality of the image and quality. It is observed from the simulation results that the PSNR value is high and MSE is low.
\end{abstract}

\section{INTRODUCTION}

Satellite images are useful for meteorology, agriculture, geology, forestry etc. These images are generally affected by absorption and scattering and this gives distortion, noise and blurriness. Enhancement of visual quality depends on increasing the resolution but excess resolution leads to blurriness.

Satellite images often have poor perceptional quality, so it is needed to improve the quality of the image. Generally to enhance images increasing the resolution is done but this does not improve the quality of the image. Using Gaussian filtering along with discrete wavelet transform and morphological filtering improves PSNR and decreases the mean square error value and thereby the quality of the image is improved. Zero padding and cyclic spinning were used to improve the perceptional quality of the image. Zero padding is a spectral analysis to improve the accuracy of reported amplitudes which are the features of the image. In this method wavelet transform of a low resolution image is taken and zero matrices are embedded to transform the image by discarding high frequency sub-bands and then inverse wavelet transform is done and thus high resolution image is obtained. In cyclic spinning initially zero padding is applied to high resolution images and after spatial shifting transforming of the image is done. Both the methods concentrate on edges and do not contribute to noise removal and preservation of noise.

This paper is organized into six sections. Section 2 reviews the literature about satellite image enhancement methods. Overview on Gaussian filtering, dwt and morphological filtering is presented in section 3. Section 4 discusses the proposed methodology. Simulation results are reported in section 5. Finally, conclusions drawn from this work are presented in section 6 .

\section{LITERATURE REVIEW}

Anumolu Lamsika[2] proposed a method for satellite image enhancement using the techniques of discrete wavelet transform and morphological filtering to improve the quality of image and thereby enhance it. Mrs.S.Sangeetha [6] proposed a method for improving the satellite images by using the noise removal and sharpening edges. Introduction to wavelet and wavelet transforms was proposed by CS.Burrus [4] which does not affect lower frequency components of the image and thus information is conserved. CC Lai [3] proposed a method in which watermarking techniques are embedded to image to prevent image processing attacks based on DWT. R.Thriveni [5] proposed a method on improving satellite images quality by using DWT and threshold decomposition along with morphological filtering.

Discrete wavelet transform is used for transforming the image in frequency domain by using the Haar transform. Morphological filtering can be done by using bicubic, bilinear and the nearest neighbor methods. They have used dwt even for encrypting the image. Wavelets basics are discussed here in these papers.

\section{OVERVIEW ON GAUSSIAN FILTERING, DWT AND MORPHOLOGICAL FILTERING}

\subsection{Gaussian Filtering}

Original image is blurred by convoluting with a Gaussian filter with a variance 0.02 and mean 0 .

Thus noise is removed from the input image after reading it, we use the fspecial function to create a gaussian filter. In higher frequencies we have noise which is blurred using a Gaussian filter and information in the lower frequencies is conserved without losing originality. 


$$
f(x, y)=\frac{1}{2 \pi \sigma^{2}} e^{-\left[\left(x-\mu_{x}\right)^{2}+\left(y-\mu_{y}\right)^{2}\right.} \mathrm{V}\left(2 \sigma^{2}\right) .
$$

\subsection{Discrete Wavelets Transform}

The image is transformed in the frequency domain. The noise exist in the high frequency components while in the low frequency we have information. DWT separates the input image into different sub band images, namely LL, LH, HL, and HH. DWT has been employed in order to preserve the low frequency components of the image. LL (low-low) has average pixel values, LH (low-high) has horizontal variation, HL (high-low) has vertical variation while $\mathrm{HH}$ (high-high) has diagonal variation. Here we use Haar transform, in which we take sum average and difference average so that on adding and subtracting them we get the initial values again. Threshold decomposition is used to approximate the values which are close to zero to it. Since an image is two dimensional we apply dwt for row and then to columns. Inverse dwt is applied to reconstruct the image again.

\section{DWT}

$$
\begin{aligned}
& a^{1}=\left(\frac{x_{1}+x_{2}}{\sqrt{2}}, \frac{x_{3}+x_{4}}{\sqrt{2}}, \cdots, \frac{x_{N-1}+x_{N}}{\sqrt{2}}\right) \\
& d^{1}=\left(\frac{x_{1}-x_{2}}{\sqrt{2}}, \frac{x_{3}-x_{4}}{\sqrt{2}}, \cdots, \frac{x_{N-1}-x_{N}}{\sqrt{2}}\right)
\end{aligned}
$$

a-sum average

d-difference average

IDWT

$$
f=\left(\frac{a_{1}+d_{1}}{\sqrt{2}}, \frac{a_{1}-d_{1}}{\sqrt{2}}, \ldots, \frac{a_{N / 2}+d_{N / 2}}{\sqrt{2}}, \frac{a_{N / 2}-d_{N / 2}}{\sqrt{2}}\right)
$$

\subsection{Morphological Filtering}

In this method, we use bicubic interpolation, first zeros are appended at the four edges twice and then pixel values are repeated twice along rows and columns by scaling. Now interpolation is done by taking an average across the square obtained by taking two elements along the left, right, top and bottom. Cubical distance is calculated for each element in the square which is taken into account while doing average. Thus interpolation is done and we smoothen the image and fill the missing gaps. $\mathrm{d}$ is difference in pixel coordinates.

$\mathrm{d}<1 ; \quad \mathrm{s}=3 / 2 * \mathrm{~d}^{\wedge} 3-5 / 2 * \mathrm{~d}^{\wedge} 2+1 ;$

$\mathrm{d}<2 ; \quad \mathrm{s}=-1 / 2 * \mathrm{~d}^{\wedge} 3+5 / 2 * \mathrm{~d}^{\wedge} 2-4 * \mathrm{~d}+2$;

$\mathrm{s}$ is the cubical distance
Demosaicing is done to improve the view ability of the image since green color is visible easily to the eye. Here we have a matrix of every alternate row consisting of alternate green and blue and the next rows of green and red being multiplied to the image and then interpolated in areas where the corresponding color value is not known.

\section{PROPOSED METHODOLOGY}

In this paper we use Gaussian filtering, dwt and morphological filtering for better results. Fig.1 shows the block diagram for Gaussian filtering, dwt and morphological filtering.

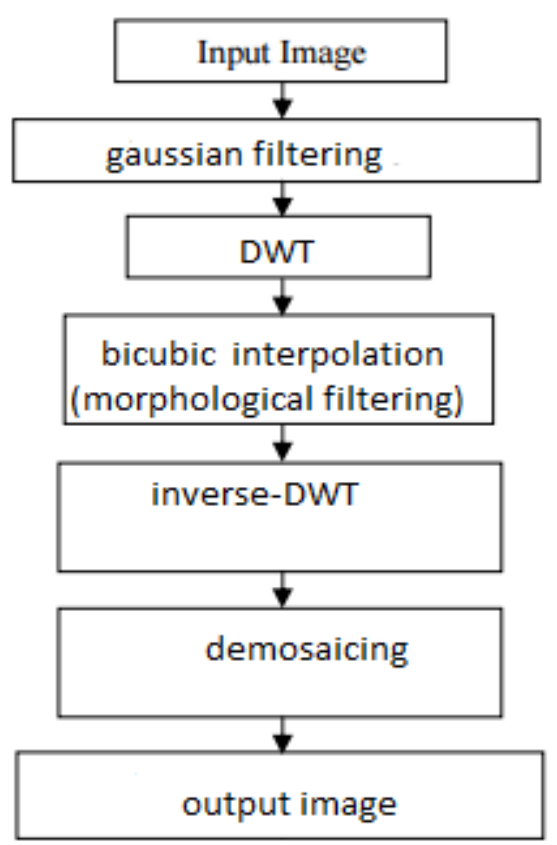

Fig.1.Block diagram of Gaussian filtering, dwt and morphological filtering

\section{SIMULATION RESULTS}

In order to verify the approach proposed in section [4] we have taken two satellite images and applied enhancement techniques on both of them and compared them. In the newer approach we have taken Gaussian filter to denoise the input image and then we apply dwt and morphological filtering. We observed the noise removal by taking the mean square error into account and the quality enhancement by the PSNR value. Here we can observe visually quality being improved and even in terms of metrics we can observe it.

\section{CONCLUSION}

Gaussian filter with dwt and morphological filtering works well in enhancing image when compared to only dwt and morphological filtering. Using dwt we transform image in frequency domain. Morphological filtering retains originality by reconstructing the image again. Gaussian filter smoothens image and removes noise thereby improving output quality. 


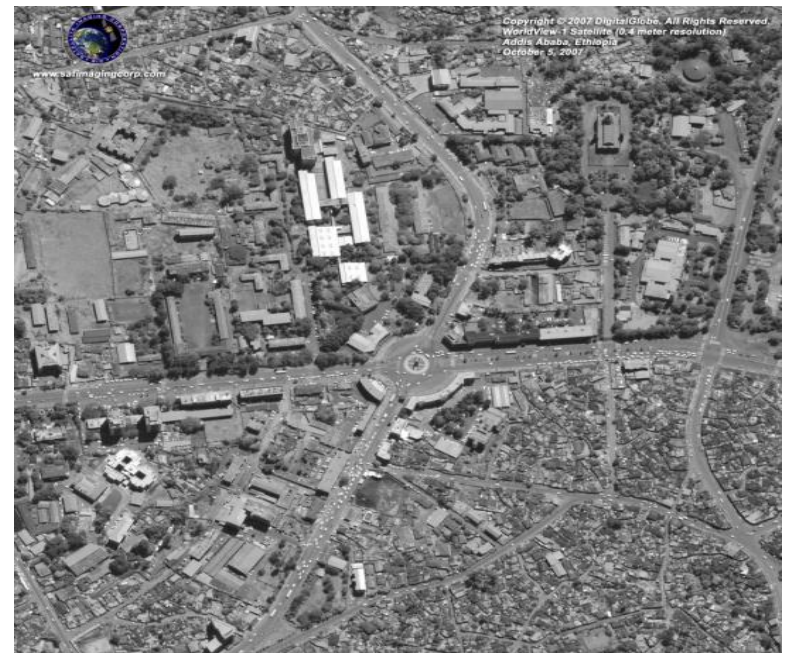

Fig.2.input test image 1

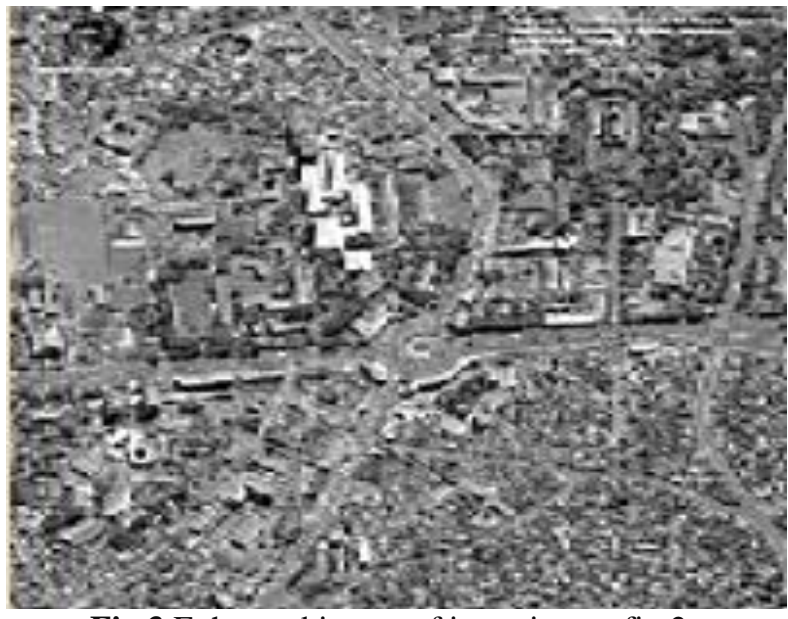

Fig.3.Enhanced image of input image fig.2.

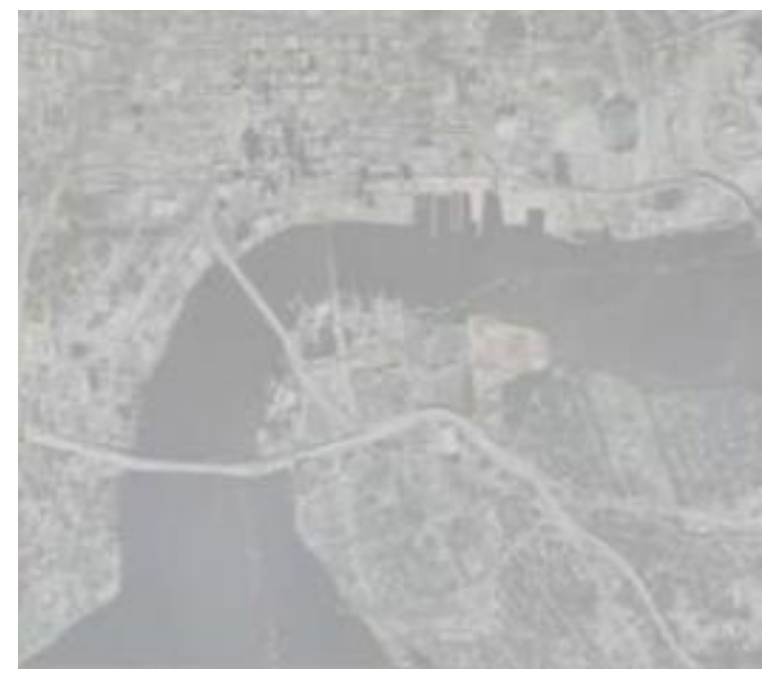

Fig.4.input test image 2

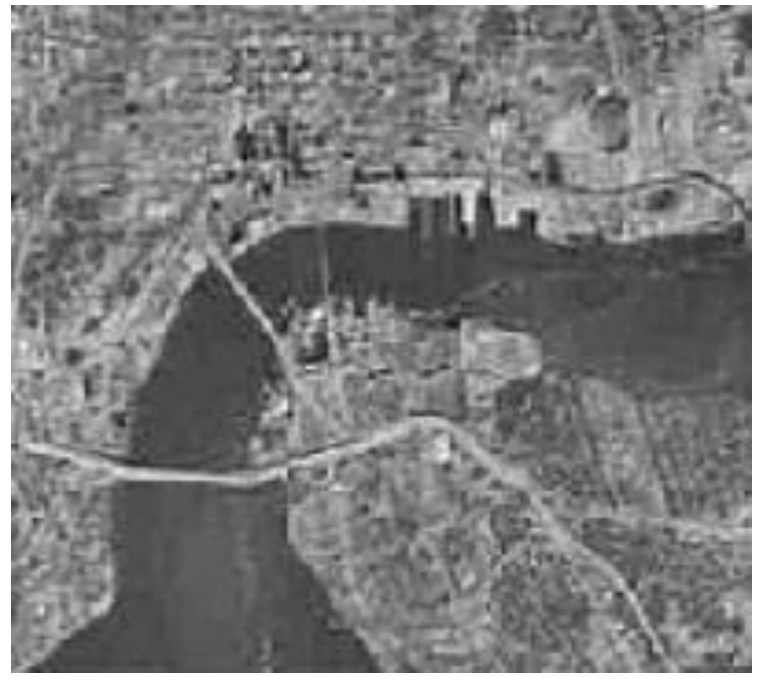

Fig.5.Enhanced image of input image fig.4

Table.1.PSNR and MSE for two input images

\begin{tabular}{|l|l|l|}
\hline & Fig- 2,3 & Fig-4,5 \\
\hline PSNR & 35.57 & 43.97 \\
\hline MSE & 9.02 & 3.00 \\
\hline
\end{tabular}

\section{REFERENCES}

[1]. Muna F. Al-samaraie "Color Satellites Image Enhancement Using Wavelet and Threshold Decomposition" IJCSI, Vol. 8, Issue 5, No 3.

[2]. Improving Quality of Satellite Image by Wavelet Transforming \& Morphological Filtering Anumolu Lasmika, K. Raveendra Vol. 3, Issue 7, July 2014

[3]. Digital Image Watermarking Using Discrete Wavelet Transform and Singular Value Decompoition, Chih-Chin Lai; Cheng-Chih Tsai IEEE Transactions on Instrumentation and Measurement ( Volume: 59, Issue: 11, Nov. 2010 )

[4]. Introduction to Wavelets and Wavelet Transforms C. Sidney Burrus, Ramesh A. Gopinath , Haitao Guo ..ISBN

[5]. Satellite image enhancement using discrete wavelet transform and threshold decomposition driven morphological filter, R.thriveni, RAMASHRI(computer communication and informatics) ICCCI, 21 February 2013.

[6]. Mrs.S.Sangeetha, Mr.Y. Hari Krishna, "Image Resolution Enhancement Technique Based on the Interpolation of the High Frequency Sub bands Obtained by DWT," International Journal of Engineering Trends and Technology (IJETT) -Volume4 Issue7-July 2013.

[7]. K.Narasimhan, V.Elamaran, Saurav Kumar, Kundan Sharma and Pogaku Raghavendra Abhishek, "Comparison of Satellite Image Enhancement Techniques in Wavelet Domain," Research Journal of Applied Sciences, Engineering and Technology. 
Ch. Haritha is studying B.Tech final year in ECE at Sreenidhi Institute of Science and Technology, Hyderabad. She has presented technical papers in various national level symposium. Her areas of interests are Digital image Processing, micro controllers, Robotics and programming languages.

B. Rupavani is studying B.Tech final year in ECE at Sreenidhi Institute of Technology, Hyderabad. She has presented technical papers in various national level symposiums. She is inventory head of TEC. Her areas of interests are Digital Image Processing, Networks, signal processing and robotics.

P. Lakshmi prasanna is studying B.Tech final year in ECE at Sreenidhi Institute of Science and Technology, Hyderabad. She has presented technical papers in various national level symposium. Her areas of interests are Robotics and digital image processing..

Dr.A.Venkataramana received his BE,ME and PHD degrees from Osmania University, Hyderabad. He has total 19 years of teaching experience. He worked as a junior telecom officer and ITS officer in the department of telecommunications for a period of 4 years. He has published various papers in IEEE, international, national conferences and journals. He is member of ISTE and ISCA. $\mathrm{He}$ has received a number of prestigious awards namely state government best lecturer in polytechnics award, ISTE best polytechnic teacher award for Andhra Pradesh state, Bharath Siksha Ratan and Rashtra Ratna award. He is presently working as faculty in the department of Electronics and Communications at Q.Q government polytechnic, old city, Hyderabad in the department of technical education, Telangana. He also published number of books in the areas of communication. His areas of interests are Digital Image Processing, Pattern Recognition, Neural Networks and Applications of Moments for Image processing Problems.

Ch.Rajendra Prasad received his BE from Bhapatla engineering college and ME from Osmania University, Hyderabad. He has total 25 years of teaching experience. He is presently working as Head of the department in the department of Electronics and Communications Engineering at Medhak government polytechnic, Telangana in the department of technical education. His areas of interests are Digital Image Processing, digital signal processing, networks and microcontrollers. 